\title{
Algunas precisiones acerca de la filosofía moral experimental de David Hume
}

\author{
Sofia Calvente \\ Universidad Nacional de La Plata \\ Facultad de Humanidades y Ciencias de la Educacion \\ Departmento de Filosofía \\ Buenos Aires \\ Argentina \\ vicentesofia@yahoo.com.ar
}

Article info

CDD: 192

Received: 16.05.2017; Revised: 2.10.2017; Accepted: 2.10.2017

DOI: http://dx.doi.org/10.1590/0100-6045.2017.V40N3.SC

Palabras clave:

Filosofía experimental

Ciencia de la naturaleza humana

Cuestiones de hecho

Reducción explicativa

\section{Keywords:}

Experimental Philosophy

Science of Human Nature

Matters of Fact

Explanatory reduction

\section{RESUMEN}

A pesar de que existe un consenso entre los estudiosos respecto de establecer una filiación entre Hume y la filosofía experimental, prevalecen importantes divergencias al momento de determinar el objeto de estudio de la "ciencia de la naturaleza humana," como así también de establecer las razones que legitiman la aplicación del método experimental a las cuestiones morales y el modo en que lo lleva a cabo. Nos proponemos esclarecer estas tres cuestiones con el fin de precisar en qué sentido debe comprenderse el experimentalismo de Hume. Respecto de la primera, sostenemos que la ciencia de la naturaleza humana no sólo comprende el estudio de la mente, sino que abarca también el de la interacción en el marco de la sociedad a lo largo de la historia. En cuanto a la segunda, consideramos que existe un fundamento ontológico común a los fenómenos morales y naturales, en tanto ambos quedan comprendidos bajo las cuestiones de hecho, lo que habilita la aplicación del mismo método de investigación a los dos. Para echar luz sobre la tercera cuestión, profundizaremos en la centralidad conferida a los hechos, entendidos como eventos epistémicamente relevantes que son procesados mediante la reducción explicativa, Finalmente, ofreceremos algunos ejemplos para mostrar cómo Hume pone en acto el método.

\begin{abstract}
Even though there is a general agreement among the scholars concerning the relationship between Hume and experimental philosophy, yet important
\end{abstract}


disagreements prevail regarding the subject of the "science of human nature," as well as the reasons that ground the introduction of the experimental method into moral subjects, and the way in which Hume does it. Our aim is to shed some light into these three items in order to specify the sense in which Hume's experimentalism should be understood. Regarding the first one, we maintain that the science of human nature not only deals with the study of the mind but also with human interaction within society through the course of history.
Concerning the second one, we consider that there is a common ontological ground to moral and natural phenomena, because both fall into what Hume calls 'matters of fact.' This, in turn is what enables him to apply the same research method to both. In order to clarify the third item, we will delve into the central role that is given to facts, which Hume understands as epistemically relevant events, which are handled through explanatory reduction, Finally, we will show through some examples how Hume uses the method.

Si bien existe un consenso generalizado entre los especialistas respecto de las intenciones de David Hume de ubicar su "ciencia de la naturaleza humana" en el marco de lo que en ese entonces era la incipiente tradición de la filosofía experimental, no se manifiesta el mismo acuerdo al momento de definir cuál es el objeto de estudio que propone, por qué considera factible aplicar un método que fue exitoso en el ámbito natural a las cuestiones morales, ni de qué manera lleva a cabo esa aplicación.

Respecto de la primera cuestión, varios intérpretes consideran que los asuntos morales de los que se ocupa Hume se refieren estrictamente a la mente, lo que los lleva a entender que el experimentalismo en el marco de su obra se limita únicamente a la introspección. No obstante, esta lectura resulta algo sesgada ya que, por un lado, se centra únicamente en el primer libro del Tratado de la naturaleza humana y no tiene en cuenta el abordaje experimentalista de

1 Es necesario aclarar que Hume entiende como equivalentes a las expresiones "filosofía moral," "ciencia del hombre" y "ciencia de la naturaleza humana" y del mismo modo las emplearemos también nosotros. Este tipo de conocimiento se refiere a cuestiones sociales y humanas. Hume comienza la primera Investigación así: "La filosofía moral o ciencia de la naturaleza humana" (EHU 1.1) es decir que considera como sinónimos a la "filosofía moral" en sentido amplio y a la ciencia que él busca cultivar. Por lo tanto, la aplicación del método experimental al ámbito moral equivale a emplear dicho método en la ciencia de la naturaleza humana. Sin embargo, cabe destacar, también, que emplea "filosofía moral" en sentido amplio para aludir a la ciencia de la naturaleza humana, y en sentido restringido, a lo que hoy denominamos "ética." 
cuestiones de índole social, política, histórica y de crítica artística y literaria que Hume lleva a cabo en sus restantes obras. Por otro lado, suele interpretarse que por el estudio de la mente Hume alude al análisis de sus propios contenidos mentales, que se consideran inaccesibles a los demás. En cuanto a la segunda y tercera cuestiones, muchos autores suelen identificar la vinculación entre Hume y el método experimental únicamente en términos de newtonianismo, ${ }^{2}$ lo que condujo a la convicción de que el filósofo tenía la pretensión de erigirse en el "Newton de las ciencias morales."3 Sin embargo, esta interpretación suele pasar por alto el hecho de que Hume considera a Newton como uno de los personajes -si bien de los más relevantes- dentro de un grupo de filósofos que concibió y llevó a cabo un modo innovador de hacer filosofía natural, el cual comenzó a desarrollarse previamente y continuó con posterioridad a su participación en él (HE 6.71, 541-542). Cuando se refiere a la "nueva corriente filosófica" (ABST, SB 645 [p. 115]), siempre lo hace aludiendo a un colectivo de autores y no a una figura en particular. Por lo tanto, entendemos que la filiación de Hume con la filosofía experimental debe estudiarse teniendo en cuenta los lineamientos generales que la caracterizan, sin la pretensión de buscar una identificación rigurosa con los postulados de Newton, ya que ese es el modo en que él mismo pretende insertarse en dicho movimiento.

En lo que sigue, nos proponemos abordar las tres cuestiones que hemos señalado, con el fin de alcanzar una comprensión más adecuada del objeto, la fundamentación y la metodología de la filosofía moral experimental de Hume. A nuestro criterio, estas cuestiones requieren de un estudio preciso, con el fin de evitar caer en lo que se ha constituido como lugares comunes en la historiografía de la Filosofía Moderna, que la vinculan estrictamente con el problema del conocimiento y definen a este último en términos de procesos mentales individuales (vd. Haakonssen, 2004, 2006). Como mostraremos a continuación, en el caso de Hume y de la filosofía experimental en general, estas características distan de ser adecuadas para delinear tanto su objeto de estudio como el modo de abordarlo.

\footnotetext{
2 Slavov (2016) es quien hace esta apreciación, con la que coincidimos plenamente.

${ }^{3}$ Este mote le fue aplicado originalmente por Passmore (1952, p. 43) y luego replicado por muchos otros intérpretes. Vd. Noxon (1973, pp. 28-30) para una breve reseña del surgimiento de este epíteto, como así también de los intérpretes que adhirieron a él y lo usaron en consecuencia.
} 


\section{El objeto de estudio de la ciencia de la naturaleza humana}

Habitualmente suele entenderse que la ciencia de la naturaleza humana que Hume se propone desarrollar se refiere únicamente al estudio de los elementos y operaciones mentales. Tal como afirma Broughton (1992, pp. 155-156), muchos comentadores consideran que Hume propone al comienzo del Tratado que el objeto de investigación de esta ciencia son las percepciones, y asumen que esas percepciones equivalen a lo que Locke llama ideas, es decir, estados de conciencia cuyos objetos inmediatos dependen de esos estados de conciencia mismos, y sólo pueden ser objetos para la propia mente que los percibe. ${ }^{4}$ Más aún, agregan que el ámbito de investigación científica para Hume son sus propias ideas lockeanas, lo que se ve reforzado por el hecho de que se rehúsa a hacer referencia a la realidad extramental para distinguir impresiones de ideas. ${ }^{5}$ Se asume que, en tanto las percepciones son contenidos de naturaleza mental, resultan accesibles únicamente al dueño de esa conciencia, porque son de índole privada. Por lo tanto, la única forma de experiencia que el científico moral podría consultar son los contenidos de su propia mente, mediante el método de la introspección.

Esta interpretación habitual genera, a nuestro juicio, diversos problemas. En primer lugar, cabría preguntarse si es pertinente atribuirle a Locke una noción de experiencia que se identifique exclusivamente con las ideas, para luego transferírsela sin más a Hume. En segundo lugar, hacer depender a la ciencia de la naturaleza humana sólo de la introspección acarrea el problema de cómo garantizar la comunicabilidad y verificabilidad de los contenidos mentales, ${ }^{6}$ cuestión que Hume no parece poner en duda. En tercer lugar, habida cuenta de que plantea que en esta ciencia debemos partir de "una observación cuidadosa de la vida humana" (T Intro, SB xix), es necesario determinar si es posible sostener que se limita únicamente a los contenidos mentales, o bien, si cabe

\footnotetext{
${ }^{4}$ Entre los autores que han asimilado la noción de experiencia humeana a las ideas de Locke se encuentran Kemp Smith (1941, p. 108), Heinemann, (1941), Rábade Romero (1973, p. 34), Stroud (1977, pp. 18-19), Flew (1985, p. 184), Dicker (1998, p. 2) y Carlin (2009, p. 159).

${ }^{5}$ Hume dice que es una diferencia que "cada uno percibirá en seguida por sí mismo" (T 1.1.1, SB 1-2), y sostiene además, que no es posible establecer una relación causal entre la realidad extramental y la mental (vd. T 1.4.2, SB 212). Vd. Everson (1988).

${ }^{6}$ Quien plantea este problema es Noxon (1973, p. 81).
} 
interpretar que se ocupa de fenómenos sociales, o ambas cosas a la vez. Finalmente, si éste último es el caso, debería esclarecerse cómo articular el ámbito mental con el extramental.

Por razones de espacio, nos limitaremos a dejar señalados los dos primeros problemas y abordaremos el tercero, para luego dedicarnos con mayor profundidad a la cuestión de la aplicabilidad del método experimental a las cuestiones morales. Sin penetrar a fondo en la discusión, nos inclinamos a pensar que Hume postula a la investigación de los contenidos mentales como la parte troncal de su proyecto -al menos en el Tratado-, en la medida en que "es evidente que todas las ciencias se relacionan en mayor o menor grado con la naturaleza humana" ( $\mathrm{T}$ Intro, SB xv), pero que esto no agota en absoluto los alcances de la ciencia que busca establecer. El estudio de lo que Hume denomina "lógica" se concentra en explicar los principios y operaciones de la mente, como así también la naturaleza de las ideas (T Intro, SB xv; ABST, SB 646), en la medida en que ese conocimiento es útil para hacer progresar a las restantes disciplinas, ya que todas están comprendidas dentro de la naturaleza humana y dependen de ella (ABST, SB 646). Tal como afirma Owen (1999, p. 66), la investigación pormenorizada de los elementos y operaciones del entendimiento que Hume lleva a cabo en el Libro I del Tratado, antes que indicar que la ciencia de la naturaleza humana consiste exclusivamente en el análisis introspectivo de los contenidos mentales, debe entenderse como un primer paso en el estudio del impacto de la nueva ciencia que Hume viene a proponer, en las restantes ciencias. Ese estudio comienza con la lógica, pero está en los planes de Hume extenderlo a las demás, como queda claro en la "Advertencia a los Libros I y II": "El lector deberá tener en cuenta, tan sólo, que no todas las cuestiones que allí [en la Introducción] me he propuesto son tratadas en estos dos volúmenes" ( $\mathrm{T}$ Adv, SB xii). De esta manera, el propio Hume es quien nos indica que debemos tomar en cuenta otras de sus obras para tener una visión acabada de la ciencia del hombre. Si bien Hume planeaba escribir ulteriores volúmenes dedicados a cuestiones estéticas y políticas, finalmente abordó esos temas en diversos ensayos (vd. Immerwahr, 1991), a los que, si tomamos en cuenta las "Advertencias," debemos considerar también para lograr una perspectiva completa de la naturaleza humana: "Si tengo la suerte de salir airoso, continuaré mi obra examinando los problemas de la moral, 
la política y la crítica de artes y letras [criticism]. Con ellos se completaría este Tratado de la naturaleza bumana" (T Adv, SB xii, las cursivas son del autor). ${ }^{7}$

Queda lo suficientemente claro, entonces, que los contenidos de la mente no son el único objeto de estudio de la ciencia de la naturaleza humana ni, por supuesto, de la filosofía de Hume, sino que, tal como él mismo lo explica en el Resumen, sólo constituyen el tema central del primer y segundo libros del Tratado, cumpliendo una función que podríamos considerar como propedéutica. Los elementos, principios operativos y máximas generales que Hume examina allí se vinculan de manera fundacional con las otras ciencias -incluso con la matemática, la filosofía natural y la religión natural ( $\mathrm{T}$ Intro, $\mathrm{SB}$ xv)- a raíz de que, para hacer ciencia, los científicos deben emplear ideas y construir razonamientos. Lo que Hume pretende es comprender la naturaleza de esas ideas y de las operaciones que se ponen en juego en los razonamientos (vd. Boehm, 2013, p. 59). De esta manera, considera que ayudará a resolver muchas de las controversias que surgen en las distintas disciplinas del conocimiento, que se deben, según su punto de vista, a una falta de comprensión de la naturaleza de las ideas que se emplean. ${ }^{8}$

Por otra parte, podemos agregar, junto con Broughton (1992, pp. 156-157, 160, 166), que el propósito de Hume no es teorizar acerca de los contenidos mentales per se, sino que éstos le interesan en tanto son el resultado de la observación de la vida humana y sirven a su vez para explicar fenómenos externos a la mente. Una vez que hemos establecido cuáles son esos elementos y principios constitutivos de la mente, lo que se logra tanto a partir de la observación del "trato mutuo de los hombres en sociedad, en sus ocupaciones y placeres" (T Intro, SB xix), como de un trabajo de introspección que nos permite analizar, clasificar, relacionar y sistematizar aquello que observamos externamente, podemos emplearlos para explicar otros fenómenos morales y para resolver muchas de las dificultades que aquejan a este tipo de ciencias.

\footnotetext{
${ }^{7}$ La cuestión de si Hume continuó con su propósito de construir un sistema completo de las ciencias basado en la ciencia de la naturaleza humana luego del Tratado es materia de discusión entre los intérpretes. Sin embargo, lo que nos interesa mostrar aquí es que el mismo Hume deja en claro que no es posible restringir el alcance de esta ciencia a las cuestiones vinculadas con el entendimiento y las pasiones, es decir, al estudio de las percepciones y las operaciones mentales.

8 Boehm (2013, pp. 60-64) ofrece ejemplos de la aplicación de esta metodología a problemas matemáticos y de la filosofía natural dentro del Tratado. Vd. además, Broughton (2004).
} 
Desde esta perspectiva, las impresiones e ideas y sus modos de asociación representan algunos de esos principios simples y generales a los que Hume busca reducir los fenómenos complejos que el filósofo moral observa (vd. Hazony, 2014, pp. 165-167). Podemos ver reflejado el ida y vuelta desde el ámbito social al mental en pasajes como éste:

De aquí se deriva igualmente la utilidad de la experiencia [experience] adquirida a través de una vida dilatada y una diversidad de ocupaciones y trato con personas para enseñar[nos] [instruct us] los principios de la naturaleza humana y regular nuestra conducta futura, así como nuestra especulación. Con esta guía nos elevamos al conocimiento de las inclinaciones y motivaciones de los hombres, partiendo de sus actos, expresiones o incluso gestos; [y de nuevo] descendemos a la interpretación de sus actos a partir de sus motivaciones e inclinaciones (EHU 8.9 [pp. 108-109]). ${ }^{9}$

El investigador de la naturaleza humana puede acceder a los principios explicativos que busca porque está dotado con una capacidad de observación que le dan la práctica de su profesión y la interacción con otros hombres. La observación externa es el punto de partida de un proceso de investigación que luego se remonta a las motivaciones, temperamentos y caracteres que pueden dar origen a las conductas que se observan a partir de un ejercicio de análisis introspectivo, que está avalado por el hecho de que la naturaleza humana es común a todos los hombres y tiene un funcionamiento regular y constante (EHU 8.7). Esto le permite al investigador comprender cuáles son los principios que pueden mover las acciones de los otros hombres, dado que, en líneas generales, son los mismos que operan en su interior también. Una vez que el filósofo moral identifica y sistematiza esas motivaciones, las adopta como principios explicativos de otras acciones semejantes que observa en el transcurso de la interacción social cotidiana.

${ }^{9}$ Como veremos luego, este pasaje expresa también el principio metodológico del análisis y la síntesis que Hume emplea en la ciencia de la naturaleza humana. Estas dos interpretaciones no se contradicen, ya que podemos considerar que el recorrido desde lo social hacia lo mental y viceversa puede identificarse como un camino que opera metodológicamente por medio del análisis y la síntesis. Vd. Demeter (2012, pp. 584585). 


\section{La reforma de la investigación de las cuestiones "morales"}

Como hemos señalado al comienzo, Hume busca insertarse dentro de lo que considera como una "nueva clase" de filosofía. Más específicamente, se propone aplicar el método experimental al ámbito moral. Lo primero que es necesario aclarar es que, en esta empresa, no es ni se considera a sí mismo un pionero. Por un lado, nos encontramos con que el mismo Francis Bacon le otorga un lugar relevante al estudio de la mente y de la interacción humana en su proyecto filosófico, delimitando una rama del conocimiento que debe ocuparse de la clasificación y descripción de diferentes caracteres y disposiciones, y proponiendo el desarrollo de historias naturales de los sentidos, las pasiones y las facultades mentales (vd. Wood, 1989, pp. 90-91). ${ }^{10}$ Esta propuesta tuvo gran incidencia en diversas figuras de lo que hoy se conoce como la Ilustración escocesa, lo que explica que filósofos morales contemporáneos a Hume, tales como Francis Hutcheson y George Turnbull, entre otros, tuvieran intenciones de desarrollar una filosofía moral experimental (vd. Broadie, 2003, pp. 62-63). ${ }^{11}$ Por otro lado, es importante enfatizar que los escoceses no entendían al método experimental en términos de newtonianismo exclusivamente, ya que compartían otros aspectos del experimentalismo que habían sido establecidos con anterioridad a la aparición de Newton en la escena científica, como la construcción de historias naturales, es decir, la descripción y clasificación de los hechos bajo estudio (vd. Wood, 1989, pp. 114-115). Además, sostenían supuestos básicos experimentalistas de carácter más general, como la centralidad conferida a eventos puntuales ocurridos en circunstancias particularizables por sobre el énfasis en los textos, el empleo de herramientas como la observación y la experimentación, y el rechazo de hipótesis meramente especulativas. Es decir que cuando Hume decide emprender su tarea, reconoce

\footnotetext{
${ }^{10}$ Wood alude puntualmente a lo que Bacon sugiere en The Advancement of Learning y De augmentis scientiarum respecto de la creación de una rama del conocimiento denominada "The culture of the mind," y a las historias naturales que delinea en Parasceve.

11 Por su parte, Rivers (2000, p. 282) señala que dentro de esta incipiente tradición de la filosofía moral experimental, Hume fue el único que, en el siglo XVIII, continuó con la propuesta de Shaftesbury de basar su teoría moral experimental únicamente en la experiencia y la observación del comportamiento humano, la sociedad y la historia, sin apelar a argumentos de corte religioso.
}

Manuscrito - Rev. Int. Fil. Campinas, v. 40, n. 3, pp. 51-86, jul.-set. 2017. 
ya la existencia de una incipiente tradición donde encuentra presentes varios de los requisitos metodológicos que él mismo se propone respetar.

El problema principal que observa consiste en que el conocimiento moral se basa enteramente en meras especulaciones o incluso fantasías que carecen de sustento en la experiencia (ABST, 646 [pp. 117-119]). Esto se debe a que la imaginación funciona de manera "laxa" o irregular, diferente de cuando opera por medio de los principios regulares y universales de asociación: la semejanza, la contigüidad y la causalidad (T 2.1.4, SB 283). Este problema, a criterio de Hume, se arrastra desde la Antigüedad y es comparable con el que aquejaba a la filosofía natural hasta que surgió el nuevo método experimental (L 1.3, 16), y que se expresaba, entre otras cosas, en la apelación a las "ficciones de sustancia y accidente," y a los "razonamientos acerca de formas sustanciales y cualidades ocultas" (T 1.3.4, SB 226).

A partir de este diagnóstico de situación, Hume entiende que, dado que la filosofía natural atravesó una situación similar a la que estaba padeciendo la filosofía moral, había buenas razones para pensar que soluciones semejantes podían funcionar. Pero lo que de manera decisiva hace posible que soluciones similares puedan funcionar en ambos casos es que los objetos de los que se ocupan tanto la filosofía natural como la moral pertenecen al ámbito de las "cuestiones de hecho" y se diferencian de las "relaciones de ideas," en tanto las primeras se arraigan en la experiencia y las segundas se basan íntegramente en operaciones mentales, "independientemente de lo que pueda existir en cualquier parte del universo" (EHU 4.1. [p. 48]). Las relaciones de ideas versan sobre entidades abstractas, como las que son objeto de la matemática. Por lo tanto, son independientes de cómo son las cosas en el mundo. Los razonamientos de este tipo están regidos por el principio de no contradicción. Por su parte, las cuestiones de hecho tratan sobre eventos que suceden en la naturaleza y en la vida mental y social de los seres humanos. Los razonamientos relativos a estas cuestiones no están regidos por el principio de no contradicción, porque lo contrario a una cuestión de hecho siempre es posible (vd. EHU 4.1-2). Estas características hacen que los razonamientos acerca de las relaciones de ideas sean necesariamente verdaderos, mientras que los inherentes a las cuestiones de hecho sólo alcancen distintos grados de probabilidad. Por lo tanto, las esferas de conocimiento no sólo son diferentes, sino en algunos puntos incluso antagónicas, lo que imposibilita transpolar el método de investigación válido para las relaciones de ideas, a las cuestiones de hecho. Es decir que aquello que habilita la unidad metodológica de lo natural y 
lo social es la unidad ontológica ${ }^{12}$ de los objetos de los que se ocupan estas disciplinas, que se definen, en ambos casos, como cuestiones de hecho.

La solución, que ha sido probada exitosamente en el ámbito natural, es sustentar la ciencia de la naturaleza humana en la experiencia. Esta alternativa parece ser el único modo de poner un freno a la propensión a guiarnos "por cualquier trivial inclinación de la imaginación" (T 1.4.3, SB 224) y a perdernos en debates que parecen no tener fin, porque restringe las especulaciones y suposiciones a aquello que es susceptible de ser formulado a partir de la experimentación y a la observación, o bien de ser probado por medio de ellas. Por otra parte, resulta de vital importancia proceder de manera sistemática y metódica tanto en la observación como en la comparación, clasificación y organización de aquello que es producto de la observación. En definitiva, Hume plantea que es posible abandonar el viejo modo de hacer filosofía moral basado en el sentimiento y el funcionamiento irregular de la imaginación, porque el ámbito moral, al igual que el natural, es pasible de racionalización, de un conocimiento sistemático y preciso, si la imaginación se conduce mediante principios universales y regulares. Pero, además, sostiene que es necesario hacerlo a partir de un método diferente del que se basa en meras hipótesis y suposiciones, que se apoye en la experiencia y la observación, a la manera en que lo hace la nueva filosofía natural experimental.

Este propósito, lejos de ser abandonado luego del Tratado, es reafirmado en Investigación sobre los principios de la moral e incluso extendido a la historia en Historia de Inglaterra. En la segunda Investigación, vemos reaparecer este enfoque cuando Hume opone el método experimental, que considera como el más adecuado para llevar a cabo investigaciones en el ámbito moral, a la "ciencia abstracta." Así, afirma que

Como esta es una cuestión de hecho y no de ciencia abstracta, sólo podemos esperar tener éxito si seguimos el método experimental y

\footnotetext{
12 Por supuesto que estamos empleando la noción de "ontología” en consonancia con el pensamiento humeano, sin tener la pretensión de referirnos a la "realidad sustancial" de los objetos que forman parte de las cuestiones de hecho, porque en el marco de la filosofía de Hume eso es imposible. Los entendemos como "objetos del pensamiento" (vd. EHU 4.1). Con la expresión "unidad ontológica” buscamos indicar que hay algo que es común a los objetos morales y naturales previo a su abordaje epistémico, que es su calidad de ser cuestiones de hecho, y que los diferencia de aquellos que pensamos mediante las relaciones de ideas.
} 
deducimos máximas generales a partir de una comparación de casos particulares. El otro método científico, el cual prescribe partir de un principio abstracto y general y luego dividirlo [branched out] en una diversidad de inferencias y conclusiones, tal vez sea más perfecto en sí mismo, pero se adapta menos a la imperfección de la naturaleza humana y es una fuente común de ilusión y error, tanto en este como en otros temas. En la actualidad los hombres están curados de su pasión por las hipótesis y los sistemas de filosofía natural y sólo atenderán a aquellos argumentos que se deriven de la experiencia. Llegó la hora de intentar una reforma semejante en todas las disquisiciones morales y rechazar todo sistema de ética que, aunque sea sutil o ingenioso, no se fundamente en hechos [facts] y observaciones (EPM 1.10 [p. 107]).

En este pasaje podemos ver claramente que el empleo de un método u otro se fundamenta en la naturaleza del objeto a ser estudiado. El éxito de la empresa está supeditado a la correcta identificación de aquello que va a ser investigado, lo que determina cuál es el procedimiento que debe seguirse en su abordaje. En este caso, Hume identifica a las cuestiones morales como cuestiones de hecho, de ahí que lo que critica es la transpolación de un método que resulta adecuado para las relaciones de ideas, al ámbito de las cuestiones de hecho, donde da por resultado ilusiones, errores y suposiciones infundadas, porque parte de la sola especulación. La transpolación resulta inadecuada porque, como hemos señalado más arriba, estos dos campos de conocimiento comprenden objetos diferentes y están regidos por principios distintos.

La historia es otra disciplina donde resulta viable la aplicación del método experimental, ya que también trata acerca del establecimiento de hechos (EHU 5.5 n8; T App, SB 624; ESY - C, 257; ESY - PA, 447; ESY - Su, 578; HE 1.1, $4 ; 2.12,3-4)$. Al igual que sucede en la investigación que se desarrolla en el ámbito natural y el moral, parte de la tarea del historiador consiste en buscar la causa principal y las causas concomitantes de los hechos, y en saber discernirlas de los detalles irrelevantes (HE 2.12, 4-5). Por lo tanto, resulta pertinente también en este ámbito adoptar una modalidad de investigación que nos permita determinar qué eventos pueden contar como casos para explicar determinados fenómenos, por medio del desarrollo de la capacidad y las herramientas para discriminar las circunstancias relevantes de las meramente accesorias. 


\section{La continuidad entre los ámbitos natural y moral}

Como acabamos de señalar, Hume rechaza la posibilidad de aplicar un método especulativo al campo de las ciencias morales, mientras que considera pertinente apelar al experimental, que probó ser exitoso en su aplicación al estudio de los fenómenos naturales. Esto lo lleva a afirmar decididamente que "al juzgar las acciones de los hombres debemos proceder basándonos en las mismas máximas que cuando razonamos sobre los objetos externos" (T 2.3.1, SB 403). Ahora bien, ¿cuál es el fundamento que justifica el empleo del método experimental en las ciencias morales?

Hume enumera una serie de rasgos que habilitan este procedimiento. El primero de ellos consiste en afirmar que existen ciertas analogías entre el plano natural y el moral desde el punto de vista epistémico, que se apoyan, como hemos indicado, en continuidades ontológicas inherentes a ambos. El segundo tiene que ver con el empleo de la misma metodología de razonamiento para procesar los datos empíricos, que ha recibido el nombre de "reducción explicativa." Por último, el tercero se vincula con la centralidad conferida a los hechos que se establecen a partir de la experiencia y la observación. En lo que sigue, revisaremos cada uno de ellos.

La primera cuestión, relativa a la vinculación entre lo natural y lo moral, se hace notar particularmente en la reflexión acerca los aspectos comunes a seres humanos y animales. Hume afirma que la estructura general de los cuerpos humano y animal es la misma o casi la misma. Por lo tanto, las mismas hipótesis que explican el funcionamiento de esa estructura en un caso, pueden explicarlo en todos. Luego extiende la analogía al plano de lo mental para comprobar que las mismas pasiones operan en ambos y que las causas que las producen coinciden también (T 2.1.12, SB 325-326), lo que implica afirmar la validez de la aplicación de esta suerte de "método," que denomina analógico, ${ }^{16}$ tanto para el plano de lo físico como para el de lo mental. ¿Cuál es el fundamento de este paralelo? Como veremos más abajo, Hume considera que el conocimiento en el ámbito de las cuestiones de hecho se apoya en los razonamientos causales. Un principio constitutivo de este tipo de razonamientos consiste en "una especie de analogía, que nos hace esperar de cualquier causa los mismos efectos que

16 Hemos entrecomillado el término "método" porque, si bien el mismo Hume lo emplea en la cita, hace un uso laxo de él que no se corresponde estrictamente con el método experimental. 
hemos observado [que] resultan de causas similares" (EHU 9.1 [p. 128]). ${ }^{17}$ Si las causas son semejantes, esperamos que den lugar a efectos semejantes. Cuanto mayor sea la semejanza, más perfecta será la analogía y más confianza podremos depositar en la conclusión que extraigamos. A partir de este principio, sostiene que "es la semejanza entre las acciones externas de los animales y las realizadas por nosotros mismos la base por la que juzgamos que sus acciones internas se asemejan igualmente a las nuestras" y de la misma manera, podemos concluir que "así como nuestras acciones internas se asemejan entre sí, también las causas de que se derivan tienen que ser semejantes" (T 1.3.16, SB 176-177).

Hume cree decididamente que el procedimiento analógico, que tiene vigencia en el plano anatómico y fisiológico, puede ser llevado "más lejos," hasta el ámbito moral (EHU 9.1 [p. 129]) porque no ve disrupciones esenciales entre ambos. Así como hemos visto que propone una continuidad entre la naturaleza humana y la animal, de la misma manera lo hace entre el plano físico y el mental, lo que valida una vez más la posibilidad de aplicar el método experimental en ambos casos. Por supuesto que la afirmación de una continuidad entre lo físico y lo mental no puede ser tomada a la ligera, sino que implica desafiar lo que muchos consideraban como verdades establecidas en ese momento. ${ }^{18}$

Antes de seguir avanzando en el tema, es necesario aclarar que la analogía respecto del funcionamiento de lo mental y lo corporal no implica necesariamente asumir que Hume sea un monista, ya que no busca eliminar las diferencias entre lo que considera que son dos categorías ontológicas diferentes, como así tampoco implica que reduzca los fenómenos mentales a los corporales, o a la inversa, como podrían hacerlo quienes adoptan una postura materialista o una inmaterialista.

Como es sabido, Hume rechaza la noción de sustancia (vd. T 1.4.3 y 1.4.5), por lo que comprende la diferencia entre lo material y lo inmaterial en términos

\footnotetext{
${ }^{17}$ Esta es una formulación de la cuarta de las "Reglas para juzgar [acerca] de causas y efectos." Al respecto, afirma que "debemos este principio a la experiencia, y es el fundamento de la mayoría de nuestros razonamientos filosóficos" (T 1.3.15, SB 173).

18 Existe una larga tradición de pensamiento que sostiene que no sólo existe una escisión entre el reino animal y la naturaleza humana, sino entre el plano mental y el corporal, tradición de la cual Descartes es uno de los representantes más emblemáticos. Vd. Buckle (2001, p. 234).
} 
de localización espacial. ${ }^{19}$ Sin embargo, debemos aclarar, por las razones que veremos a continuación, que la distinción material/inmaterial no coincide con la diferencia entre cuerpos y mentes (vd. Guerrero del Amo, 2001, p. 59). Una entidad material es aquella capaz de conjunción local con otras entidades; es extensa o compone objetos extensos. Una entidad inmaterial es incapaz de localización espacial, por lo que no es extensa ni puede componer extensión por medio de conjunción local. ${ }^{20}$ Esta diferencia se expresa cuando Hume distingue entre percepciones extensas, como las impresiones de color y solidez, y percepciones no extensas, como las de reflexión, o las impresiones olfativas y gustativas (vd. T 1.4.5, SB 235-236). Es decir que una percepción es o bien de naturaleza material, si tiene localización espacial, o bien inmaterial, si no la tiene, pero en ningún caso puede ser ambas cosas al mismo tiempo. Por lo tanto, no todo lo que existe es extenso ( $\mathrm{T}$ 1.4.5, SB 235), ya que hay percepciones que existen sin tener localización alguna ni necesitan existir en o conjugarse con entidades materiales para existir. ${ }^{21}$ Más aún, Hume considera que las percepciones espaciales no pueden conjugarse localmente con las no espaciales. Por ejemplo, si bien todos estaríamos dispuestos a admitir que un higo y una aceituna se diferencian por su sabor, no podemos afirmar que lo dulce del primero y lo amargo de la segunda se encuentran "dentro del cuerpo visible mismo" de dichos frutos (T 1.4.5, SB 236).

Llegados a este punto, debemos destacar varias cuestiones importantes. En primer lugar, es necesario tener presente que todas las percepciones son de naturaleza mental (T 1.4.2, SB 212; vd. 2.2.6, SB 366; 3.1.1, SB 456; EHU 12.12), por lo que, dada la distinción entre percepciones extensas e inextensas, la mente admitiría entidades de ambos tipos. En segundo lugar, no estamos en

19 Por ese motivo, Pitson (2002, pp. 54-55) sostiene que Hume rechaza el dualismo como un tipo de teoría de la sustancia (al igual que rechaza el no-dualismo de Spinoza en los mismos términos), conservando la distinción entre aquello que está ubicado espacialmente y aquello que no, como veremos más abajo.

${ }^{20}$ Estamos siguiendo la distinción propuesta por Cummins (1995, p. $50 \mathrm{n} 12$ ), aunque con la salvedad de que no compartimos su identificación de lo material con lo físico y lo inmaterial con lo mental, por las razones que mencionaremos a continuación. Como se habrá notado, Hume entiende que materia y extensión son la misma cosa (vd. T 1.2.5, SB 65).

${ }^{21}$ Podemos recordar aquí el principio de separabilidad, mediante el cual Hume afirma que una percepción, sea de la naturaleza que sea, es distinguible de cualquier otra y puede existir separadamente de cualquier otra cosa. 
condiciones de asociar lo sensible a lo extenso y lo inteligible a lo inextenso, porque el ámbito de lo sensible está compuesto por elementos extensos e inextensos, como en el caso de la forma, la textura y el sabor de un higo. Lo mismo puede decirse del plano inteligible: las ideas no son únicamente inextensas, sino que puede haber ideas extensas, por ejemplo, aquellas que representan objetos espaciales. Así, Hume afirma que "la idea misma de extensión no está copiada sino de una impresión y en consecuencia tiene que corresponder perfectamente a ésta. Decir que la idea de extensión corresponde a algo es decir que es extensa" (T 1.4.5, SB 239-240; vd. T 1.2.3, SB 34; 1.4.5, SB 232-233). ${ }^{22}$ Por lo tanto, no es posible caracterizar los cuerpos y las mentes en términos de sustancias materiales e inmateriales respectivamente, de manera exclusiva.

Por otra parte, Hume afirma que, "aunque un objeto extenso no sea susceptible de conjunción local con otro que existe sin estar en ningún lugar y sin extensión, con todo les es posible establecer muchas otras relaciones" ( $T$ 1.4.5, SB 237). Es decir que descartar la posibilidad de conjunción local entre lo extenso y lo inextenso no supone adscribirles incompatibilidad en otros planos. Así, admite que pueden entablar relaciones causales y de contigüidad temporal, entre otros modos de conjugación posibles. ${ }^{23}$ Esto se debe a que, al rechazar la teoría de la sustancia, no hay nada que impida la posibilidad de la coexistencia de dos propiedades de naturaleza diferente -siempre y cuando se excluya la conexión local-, como parte de un haz de percepciones interconectado causalmente. Por lo tanto, puede afirmarse que en la mente y en el cuerpo existen elementos mixtos -extensos e inextensos- vinculados entre sí de diversas maneras. ${ }^{24}$

\footnotetext{
22 Norton y Norton (2007, p. 483) hacen notar que Hume sostiene este "inusual punto de vista" respecto de que algunas de nuestras percepciones son extensas (la traducción es nuestra). Sobre el tema, vd. Flage (1990, p. 112) y Anderson (1995).

${ }^{23}$ Cummins (1995, p. 51) observa que la admisión de esta posibilidad por parte de Hume lo llevó inclusive a modificar su definición inicial de causalidad (T 1.3.2, SB 7478), la que incluía, entre otros, los requisitos de la contigüidad espacial y temporal, abandonando el primero de ellos. El mismo Hume anticipa este cambio cuando presenta por primera vez los elementos que componen la relación causal ( $\mathrm{T}$ 1.3.2, SB 75). Luego, en la primera Investigación, deja de lado definitivamente el requisito de la contigüidad. Vd. Slavov (2016, p. 290).

${ }^{24}$ Por supuesto que la postura de Hume respecto de lo físico y lo mental, lo extenso y lo inextenso, no está exenta de problemas, entre los cuales se cuentan la dificultad de
} 
Como hemos indicado anteriormente, Hume plantea que existe una interacción causal entre los planos mental y físico en ambos sentidos. Más allá de las resistencias filosóficas que pudiera despertar esta afirmación, sostiene que la interacción es una cuestión de hecho que se establece a partir de la observación. Esto es posible, en principio, porque en el plano de las cuestiones de hecho, "considerando el asunto a priori, cualquier cosa puede producir cualquier otra" (T 1.4.5, SB 247; vd. 1.3.15, SB 173). Es decir que no hay ninguna restricción que impida, por ejemplo, que lo corporal pueda ser causa de estados mentales, en tanto, "podemos concluir con certeza que el movimiento puede ser, y de hecho es, causa del pensamiento y la percepción" ( $T$ 1.4.5, SB 248 [p. 393]; vd. 1.4.5, SB 250). Por otra parte, "es una cuestión de experiencia común [common experience] que el movimiento de [nuestros miembros] sigue el mandato de la voluntad, como otros movimientos naturales" (EHU 7.15 [p. 91]). ${ }^{25}$ Podemos ver, entonces, que la acción causal del cuerpo sobre la mente y de ésta sobre aquel se toma en los mismos términos: es un hecho que se establece por medio de la experiencia.

Llegados a este punto, podemos comprender de qué manera las analogías o semejanzas estructurales que Hume emplea metodológicamente para conocer la naturaleza animal a partir de la humana, y la interacción entre lo mental y lo corporal, tienen por fundamento una continuidad, es decir una ausencia de disrupción, de carácter ontológico entre los ámbitos natural y moral. Sin embargo, como se infiere a partir de lo que hemos visto en este apartado, esa continuidad ontológica no implica una homogeneidad o monismo sustancial, sino que Hume se esfuerza por mantener las diferencias entre lo físico y lo mental, y entre lo extenso y lo inextenso. La continuidad implica, entonces, que existe una interacción posible entre las entidades físicas y mentales, y que esa interacción se basa en el hecho de que, en tanto dichas entidades son cuestiones de hecho, están regidas por las mismas leyes, que son de índole causal.

mantener la distinción entre la mente y el cuerpo si no es en términos de extenso/inextenso, o la accesibilidad epistémica a los cuerpos en general, habida cuenta de que todas las percepciones son de índole mental (T 1.2.6, SB 67-68; EHU 12.12). Vd. Pitson (2002, pp. 60-61).

${ }^{25}$ Inclusive, en el ensayo "De la inmortalidad del alma," Hume llega a afirmar que cuerpo y mente están tan estrechamente conectados, que toda alteración que se observa en uno está acompañada por una alteración proporcional en el otro. De manera que debemos concluir, por las reglas de la analogía, que a la disolución de uno de ellos le sigue la disolución total del otro. Vd. ESY - IS, 596. 


\section{Paralelismos y vinculaciones causales}

Una vez que hemos hecho estas aclaraciones, resulta más fácil comprender desde qué perspectiva Hume sostiene la continuidad entre el plano natural y el moral, la que amerita aplicar los mismos principios metodológicos a los dos. Podemos explicar de manera análoga el funcionamiento de ambos empleando el principio de asociación causal. Pero, además, es posible establecer una vinculación entre ellos por medio de la causalidad, como quedó ejemplificado en el caso de la interacción entre el cuerpo y la mente. Veamos con más detenimiento lo que ambas afirmaciones implican.

En primer lugar, Hume indica que la naturaleza ha instaurado la misma economía en el plano natural y en el mental, ratificando así la analogía en cuanto a su modo de funcionamiento:

Igual que la naturaleza ha proporcionado a los cuerpos ciertos apetitos e inclinaciones a los que modifica o hace aumentar o disminuir según la situación de los fluidos o los sólidos, de la misma manera ha procedido con la mente. Según que estemos poseídos de amor u odio, surge en la mente el deseo de felicidad o desgracia de la persona objeto de esas pasiones, y de la misma forma varía con cada variación de las pasiones opuestas (T 2.2.6, SB 268).

Los principios análogos de funcionamiento no sólo se evidencian en el correlato entre lo físico y lo mental, sino también entre los fenómenos naturales y los sociales. Así, en el ensayo "De la balanza comercial," Hume compara el equilibrio de la balanza comercial con la estabilidad del nivel del agua, ya que ambos operan con mecanismos de autorregulación (ESY - BT 312-313 [p. 288]). En el mismo ensayo, además, menciona que existe una "atracción moral que surge de los intereses y pasiones de los hombres y es igual de potente e infalible" que la "atracción física" (ESY - BT 313 [p. 289]). En este caso, la atracción no actúa a nivel de los contenidos mentales, como ocurre con la que Hume menciona al caracterizar la asociación de ideas (T 1.1.4, SB 12-13), sino que se vincula con la relación entre los motivos y acciones de los integrantes de distintos pueblos.

Otro principio tomado del ámbito natural que puede aplicarse por analogía al social es el de cohesión: "Debemos ciertamente admitir que la cohesión de las partes de la materia surge de principios naturales y necesarios (...) Y por similar razón tendremos que admitir que la sociedad humana se basa en 
principios similares," ya que es posible observar una "inclinación universal" que lleva a los hombres a buscar la compañía de los demás (T 2.3.1, SB 401-402). Así, la tendencia gregaria de los seres humanos puede equipararse explicativamente a la tendencia de las partículas de materia a permanecer unidas.

Estas y otras observaciones llevan a Hume a afirmar que existe la "misma uniformidad y regularidad" en "la mutua acción de los elementos y poderes de la naturaleza" que entre los motivos y acciones de los hombres, porque en ambos casos, "causas similares, siguen produciendo efectos similares" (T 2.3.1, SB 401, vd. EHU 8.15). La constancia y regularidad en la relación entre causas y efectos, que se comprueba en ambos ámbitos por igual, es lo que determina al entendimiento a inferir la existencia de las unas a partir de los otros (T 2.3.1, SB 404, vd. EHU 8.16) y es lo que produce en "las disquisiciones morales una convicción tan fuerte como en las naturales" (EPM 6.6 [p. 191]). Esta postura puede identificarse con lo que algunos autores han denominado como el "principio de la homogeneidad," porque supone que la noción de causa debe entenderse del mismo modo al aplicarse a los eventos físicos y a los mentales (vd. Wade, 1994; Crane, 1995, p. 219; Pitson, 2002, p. 58).26 En el caso de Hume, la causalidad, tanto en el plano natural como en el moral, no está constituida más que por la observación de una conjunción constante entre tipos de objetos y una determinación mental a pasar de uno a otro. Por ende, no hay nada que indique que no deba entenderse en los mismos términos en ambos casos, en tanto lo natural y lo moral pertenecen al dominio de las cuestiones de hecho (vd. Schliesser, 2009, pp. 193-195).27 Todas las cuestiones de hecho tienen la misma estructura causal, no hay prioridad de las causas físicas por sobre las morales, ni a la inversa, porque "no hay sino un solo tipo de causa" y "la distinción corriente entre necesidad moral y física no tiene fundamento alguno en la naturaleza" (T 1.3.14, SB 171, las cursivas son del autor).

\footnotetext{
26 Puntualmente, Crane afirma que lo que pone de manifiesto el "principio de la homogeneidad" es que lo que es de índole "mental" o "física" son los términos relacionados, no la relación causal en sí.

${ }^{27}$ Schliesser sostiene que esto prueba que Hume no es un reduccionista, es decir que no reduce la causalidad moral a la causalidad física entendida en términos mecanicistas.
} 
En definitiva, para que haya ciencia, debe ser posible establecer regularidades. ${ }^{28}$ Esto es lo que permite construir explicaciones generales a partir de hechos particulares, explicaciones que luego pueden aplicarse a nuevos fenómenos singulares, lo que constituye un procedimiento característico de la filosofía natural experimental. Este modo de proceder es el que debe situarse en la base de la filosofía moral, porque en ella también es posible constatar regularidades, las que ameritan el mismo tratamiento preciso y metódico que las establecidas en la filosofía natural. Hume lo sostiene claramente en la conclusión de Una disertación sobre las pasiones:

Es suficiente para mis propósitos si he demostrado que, en la producción y conducta de las pasiones, hay un cierto mecanismo regular, que es susceptible de una disquisición exacta, igual que las leyes de la dinámica, óptica, hidrostática o de cualquier parte de la filosofía natural (OP 1. 181 [p. 153]).

En segundo lugar, Hume no sólo afirma que es posible comprender mediante el mismo principio causal lo que sucede en el plano físico y el moral, sino que la causalidad atraviesa y vincula a ambos. Es decir que además de un paralelismo, hay una interconexión entre los fenómenos físicos y los morales, lo que a su vez ratifica la homogeneidad del principio causal en el ámbito de las cuestiones de hecho. Así, sostiene que la evidencia natural y la moral se "cementan" entre sí, y respecto de ellas "nos formamos una sola cadena de argumentos", por lo que "no podemos sentir ya reparo alguno en admitir que ambas son de la misma naturaleza y se derivan de los mismos principios" ( $\mathrm{T}$ 2.3.1, SB 406; vd. EHU 8.19). Es perfectamente posible construir cadenas causales compuestas por eventos naturales y acciones voluntarias, ya que la mente no siente diferencia al pasar de unos a otros (T 2.3.1, SB 407; vd. EHU 8.19), porque ambos pertenecen al espectro de las cuestiones de hecho.

\section{La reducción explicativa}

${ }^{28}$ De Pierris (2006, p. 311) hace notar que a pesar de que, desde un punto de vista escéptico, Hume considera que el principio de uniformidad de la naturaleza no está justificado ni a priori ni inductivamente, lo considera como la mejor explicación posible y como la perspectiva que se adopta tanto en la vida cotidiana como en el ámbito científico. 
Pasemos ahora a revisar el segundo de los principios que habilitan a la aplicación del método experimental a los ambos ámbitos natural y moral, que consiste en el empleo de la misma metodología de razonamiento para procesar los datos empíricos, que ha recibido el nombre de "reducción explicativa." Demeter $(2012$, pp. 578, 582) sostiene que a la hora de comprender en qué consiste el método experimental de Hume, debemos tener en cuenta que, en el subtítulo del Tratado, Hume habla de un "método experimental de razonamiento." Con esto, quiere enfatizar que lo que es experimental no es tanto el modo en que Hume produce los datos empíricos como la manera en que procesa esos datos. Si bien puede ser cuestionable la primera afirmación, veamos en qué consiste ese método de procesamiento de los casos observados para posteriormente discutir, en el próximo apartado, si la recolección de datos puede considerarse propiamente experimental o no.

En la primera Investigación, Hume sostiene que el filósofo moral trata a los fenómenos sociales, tales como guerras, revoluciones e intrigas, de la misma manera que el filósofo natural trata a las plantas, minerales y demás elementos que constituyen su objeto de estudio. ¿Cuál es ese tratamiento? Según lo menciona en ese mismo pasaje, se trata de fijar principios generales a partir de "colecciones de [experimentos]" (EHU 8.7 [p. 108]). Este método ha recibido el nombre de "reducción explicativa," y se asocia con el del análisis y la síntesis (vd. Hazony, 2014, pp. 161-167; Demeter, 2012, pp. 584-587 y 2014, pp. 180189). El método consiste básicamente en partir desde un conjunto de casos observados que se comparan entre sí para encontrar analogías entre ellos, las que se adscriben a causas semejantes. Así se obtienen generalizaciones, que nuevamente se comparan y agrupan en base a su semejanza, para extraer principios legaliformes de mayor generalidad. El propósito reside en obtener la menor cantidad de principios que posean la mayor capacidad explicativa posible. Una vez establecidos esos principios, se los aplica a nuevos fenómenos que se estiman semejantes a los casos sobre los que se establecieron dichos principios, para explicarlos.

Al aplicar este método al ámbito moral, Hume establece ciertos principios de la naturaleza humana tales como la simpatía o el sentido moral, junto con ciertos modos típicos de operar de la imaginación, como el principio de la galera, los principios de asociación regulares e irregulares, o los modos de razonamiento probable y demostrativo. Así, reduce explicativamente una variedad de fenómenos observados y observables en los acontecimientos históricos, la interacción social cotidiana y la reflexión individual a principios 
regulares subyacentes, de la misma manera en que, en la filosofía natural, los diversos fenómenos de la naturaleza se explican por medio de principios que se infieren a partir de la experiencia, o pueden confirmarse por medio de ella (EHU 4.12 [p. 53]).

Podemos encontrar a lo largo de la obra de Hume reiteradas alusiones a esta metodología de razonamiento, algunas de las cuales han aparecido en pasajes que hemos citado más arriba (vd. EPM 1.10; HE 2.12, 3-4). ${ }^{29}$ Tal vez una de las más claras sea ésta:

$\mathrm{Si}$, al examinar varios fenómenos, descubrimos que se resuelven en un solo principio y podemos conducir este principio hasta otro, llegaremos al final a esos pocos y simples principios de los que los demás dependen. $\mathrm{Y}$ aunque nunca podamos llegar a los últimos principios, es una satisfacción ir tan lejos como nos permiten nuestras facultades (ABST, SB 646 [p 117]).

En este pasaje queda claramente explicitada la primera parte del método, es decir el camino del análisis. Al mismo tiempo, se pone de manifiesto una máxima metodológica que consiste en que los principios sólo han de ser formulados hasta donde pueden llegar nuestras facultades cognoscitivas, ya que las últimas cualidades originarias tanto de la naturaleza en general, como de la naturaleza humana en particular, nos son inaccesibles ( $T$ Intro, SB xvii; vd. EPM 5.17 n19) y cualquier afirmación al respecto caería en el terreno de las hipótesis injustificables. Luego de formulados dichos principios generales, se recorre el camino de la síntesis:

Con esta guía nos elevamos al conocimiento de las inclinaciones y motivaciones de los hombres, partiendo de sus actos, expresiones o incluso gestos; [y de nuevo] descendemos a la interpretación de sus actos a partir de sus motivaciones e inclinaciones (EHU 8.9 [p.109]). ${ }^{30}$

${ }^{29}$ Encontramos otras alusiones a este método de razonamiento en T 2.1.4, SB 282; 2.3.9, SB 441; ESY - C, 254; EHU 1.15.

${ }^{30}$ En esta ocasión, la alusión de Hume al camino de la síntesis se refiere a cuestiones morales porque en ese momento está hablando de dichos temas, pero queda claro que este camino se aplica también a las cuestiones naturales, ya que, como hemos visto recién, el filósofo moral trata a los hechos humanos de la misma manera que el filósofo natural trata a los fenómenos de la naturaleza. 
Por supuesto que, como hace notar Demeter (2012, p. 586), los principios explicativos tienen un carácter tentativo y provisorio, es decir que contienen un grado inevitable de incerteza y falibilidad, en tanto se trata de generalizaciones inductivas y no de axiomas. No obstante, estas son las características del conocimiento que podemos obtener en el ámbito de las cuestiones de hecho, donde lo contrario a cualquier caso observado es posible. Sin embargo, como vimos más arriba, la regularidad que se observa tanto en los fenómenos naturales como en los morales es lo que garantiza la posibilidad del establecimiento de dichos principios.

Tal vez el producto más acabado de la reducción explicativa sean las ocho "reglas para juzgar [acerca] de causas y efectos" que Hume establece en la sección XV de la tercera parte del Libro I del Tratado. Estas reglas se aplican tanto a cuestiones morales como naturales, porque pretenden sistematizar en unos pocos principios la operación regular del principio causal, que es aquel por medio del cual damos cuenta de todo lo que sucede en el ámbito de las cuestiones de hecho. ${ }^{31}$ Como él mismo lo afirma, estas reglas no son más que explicitaciones y ampliaciones de "los principios naturales de nuestro entendimiento" (T 1.3.15, SB 175 [p. 304]), es decir, de los principios naturales de asociación.

\section{La centralidad de los hechos}

Llegamos así al tercer principio metodológico inherente a los ámbitos natural y moral, que se vincula con la centralidad conferida a los hechos que se establecen a partir de la observación filosófica. Como señalamos al comienzo del apartado anterior, Demeter sostiene que lo propiamente experimental en la filosofía de Hume es el método de razonamiento antes que el modo de recolección de los datos empíricos. A criterio de este autor, "si el método de Hume para estudiar la naturaleza humana se apoyara sólo en la observación, sería difícil ver cómo podría merecer el rótulo de 'experimental"' (2012, p. 579)..$^{32}$ Veamos si es posible sostener esta afirmación o no.

31 Encontramos algunos ejemplos de la aplicación de estas reglas en T 1.3.12, SB 136; 2.1.3, SB 282; 3.3.1, SB 578; 3.3.1, SB 580.

32 La traducción es nuestra.

Manuscrito - Rev. Int. Fil. Campinas, v. 40, n. 3, pp. 51-86, jul.-set. 2017. 
En primer lugar, es necesario destacar, junto con Shapin (1998, pp. 3-4), que no es posible afirmar que en el siglo XVII existiera una única entidad coherente que pueda denominarse "método científico." Sí es posible reconstruir una serie de prácticas culturales que se proponían comprender, explicar y controlar el mundo natural, las que en muchos casos tenían ciertos puntos en común y también importantes divergencias. Esto hace difícil aceptar sin más la afirmación de Demeter, ya que, si bien en diversas oportunidades Hume considera como una "desventaja" no poder realizar experimentos en sentido estricto -es decir, como hechos producidos deliberadamente- dentro del ámbito moral (T Intro, SB xviii-xix), no obstante existen otras disciplinas que tampoco pueden hacer uso de la experimentación y no por ello son excluidas de la filosofía experimental. El caso más evidente es el de la astronomía, donde resulta imposible forjar experimentos, aunque sí pueden llevarse a cabo observaciones puntuales y registrarlas sistemáticamente, conforme a lo requerido en el marco del experimentalismo.

En segundo lugar, tampoco existe un acuerdo acerca de cuál es el significado de la noción de "experimento" en los siglos XVII y XVIII. Sobre todo en el siglo XVII aún coexistía un sentido más ligado a la tradición aristotélico-escolástica de registro de lo que sucede en el curso habitual de la naturaleza, con el de la producción de un hecho puntual que tiene lugar en un momento y lugar determinados, y supone la intervención del hombre para alterar ese curso regular. ${ }^{33}$ De hecho, la noción baconiana de "experimento" incluye a ambos -observación y provocación artificial-, aunque resalta como característica definitoria que se trata de hechos puntuales buscados deliberada y metódicamente por el investigador y establecidos de manera confiable, antes que de enunciados generales basados en lo que supuestamente todos pueden observar respecto del curso natural (vd. Manzo, 2001).

Finalmente, podemos agregar que en el proyecto de construcción de historias naturales de la Royal Society estaban comprendidos tanto el ámbito natural, que abarcaba las obras de Dios; como el moral, que incluía fenómenos

${ }^{33}$ Esto se ve reflejado en los diccionarios y léxicos filosóficos de la época. Vd., por ejemplo, Chauvin (1692, p. 217, col. 1) y Chambers (1728, vol. 1, p. 368, col. 1-2). Por otro lado, Dear (2006, pp. 114-115) agrega también que al menos hasta entrado el siglo XVI, el término experimentum se usaba para designar un tipo de recetas médicas en las que la prescripción del medicamento estaba avalada por la experiencia del médico que lo indicaba.

Manuscrito - Rev. Int. Fil. Campinas, v. 40, n. 3, pp. 51-86, jul.-set. 2017. 
humanos, tales como costumbres, formas de gobierno e intercambio, e incluso, aspectos de fisiología y medicina. Todos ellos podían considerarse como cuestiones de hecho y, por lo tanto, incluirse como partes de las historias naturales (vd. Shapiro, 2000, p. 114). Como señalamos más arriba, en consonancia con estos lineamientos, diversos filósofos morales se abocaron al estudio de los fenómenos mentales y sociales valiéndose tanto de la construcción de historias naturales como de la inducción, ya que concebían que existía una unión estrecha entre lo natural y lo moral (vd. Wood, 1989, pp. 95, 114-115).

Estas breves indicaciones nos muestran, por un lado, que no es posible sostener una definición única de experimentalismo. Por otro, que el método experimental no plantea como condición excluyente a la experimentación en sentido estricto. Por último, que tampoco se ocupa de manera primordial de cuestiones naturales, sino que se encuentra abierto, desde sus comienzos, a la incorporación de cuestiones humanas y sociales. Por lo tanto, consideramos que para pensar en las características relevantes del método experimental se debe poner el acento, antes que en el experimentalismo en sentido estricto, en el establecimiento confiable de hechos particulares por medio de la experiencia respetando un método específico.

Lo importante para ubicar a un filósofo dentro del marco experimentalista es entonces que las explicaciones que ofrezca sobre los fenómenos que estudia -sean éstos naturales o morales- no apelen ni a suposiciones acerca de poderes o cualidades ocultas ni a una deducción lógica a partir de principios axiomáticos, es decir, a métodos puramente especulativos, como así tampoco que recurra únicamente a las opiniones de autoridades, sino, sobre todo, a hechos concretos que sean constatables mediante la experiencia, lo que implica la puesta en práctica de la observación y/o la experimentación, según las posibilidades del caso. Siguiendo esta línea, Hume sostiene que los hechos tienen un carácter incontrovertido (vd. DNR 10.199) y son lo opuesto a una suposición arbitraria o "hipótesis abstrusa que nunca puede hacerse inteligible, ni cuadrar con ningún caso o ejemplo particular" (EPM App 1.10 [p. 258]). Por otra parte, también los que entiende, en consonancia con el nuevo tipo de filosofía, como eventos particulares que ocurrieron en un momento y lugar determinados, y que se establecen por medio de la experiencia: "Con respecto a la experiencia pasada, sólo puede aceptarse que da información directa y cierta de [aquellos] objetos de conocimiento [precisos], y de aquel [preciso] período de tiempo abarcado por su acto de conocimiento [cognizance]" (EHU 4.16 [p. 56], la 
cursiva es del autor, vd. EHU 4.20). Más allá de que esos hechos hayan sido establecidos como resultado de la producción artificial [contriving] o la recolección [gleaning up], lo importante es el rol central que, para Hume, la experiencia ocupa en este proceso, que es la misma que le conceden los experimentalistas. En este sentido, comparte con ellos el considerarla como una "autoridad" (T Intro 10, SB xviii; EHU 4.20) equivalente o incluso superior a la de las obras de los grandes filósofos. Los hechos establecidos mediante la experiencia tienen la última palabra en las investigaciones acerca de la filosofía natural, como así también en las que se llevan a cabo en la ética, la política y la historia (ESY - IP, 43-44; vd. EPM 5.6).

Por este motivo, nos parece adecuada la definición propuesta por Buckle (2001, pp. 82-83), para quien los experimentos en el marco de la filosofía humeana deben entenderse como observaciones cuyo carácter experimental consiste en el hecho de que son relevantes y lo suficientemente precisas como para fundamentar y/o probar enunciados generales sobre aquello que es objeto de investigación. Es el lector quien debe juzgar si cumplen con esa función o no, a partir de la posibilidad de verificar lo que se afirma. Por supuesto que esto no supone plantear que la experiencia sea transparente y nos permita acceder a las cualidades últimas de los objetos de conocimiento, sean éstas físicas o mentales, sino que nos proporciona un tipo de conocimiento imperfecto y limitado, pero comunicable. ${ }^{34}$ Sólo cabe agregar a la definición de Buckle que la noción humeana de experimento no comprende únicamente observaciones directas, sino también testimonios de terceros (EHU 10.5-8), ejemplos tomados de la vida cotidiana ( $\mathrm{T}$ 1.3.8, SB 99-10; EHU 5.15), experimentos "psicológicos" propuestos por el mismo Hume (T 2.2.2, SB 332), y también hechos históricos debidamente establecidos (EHU 8.7, ESY - CL, 88). Sea cual fuere la índole de los experimentos o casos, se consideran como tales por su carácter de eventos que desempeñan un papel epistémico relevante.

\footnotetext{
${ }^{34}$ Buckle (2001, p. 86) se refiere a esta limitación como la "interpretación escéptica de la experiencia," y lo señala como uno de los aspectos que marcan la influencia de Newton en Hume.
}

Manuscrito - Rev. Int. Fil. Campinas, v. 40, n. 3, pp. 51-86, jul.-set. 2017. 


\section{Algunos ejemplos}

Para finalizar, nos interesa mostrar brevemente mediante algunos ejemplos de qué manera Hume aplica el método experimental a las cuestiones morales. En la Investigación sobre los principios de la moral encontramos un pasaje donde define sintéticamente los pasos a seguir, sobre los que nos hemos explayado más arriba:

La hipótesis que nosotros abrazamos es clara. Sostiene que la moralidad está determinada por el sentimiento. Define la virtud como cualquier cualidad o acción mental que proporciona al espectador el sentimiento agradable de aprobación, y al vicio como lo contrario. Luego procedemos a examinar una cuestión de hecho clara, a saber, qué acciones tienen esta influencia; consideramos todas las circunstancias en las cuales estas acciones coinciden y, a partir de allí tratamos de extraer algunas observaciones generales con respecto a estos sentimientos (EPM App. 1.10 [p. 258]).

Concretamente, el procedimiento consiste, entonces, en comenzar por una hipótesis o afirmación que, a diferencia de aquellas de carácter puramente especulativo, sea "conforme a la experiencia," (ESY - RP, 135 [p. 146]) es decir, susceptible de comprobación mediante casos puntuales. A continuación se examinan los casos en cuestión, para establecer puntos en común que permitan formular enunciados de carácter general. Finalmente, esto conduce al establecimiento o a la justificación de la afirmación inicial, la que a su vez podrá ser empleada para explicar nuevos casos similares.

Como hemos indicado anteriormente, los casos no sólo consisten en eventos u acciones observados directamente por el investigador, sino que también se aceptan, con el mismo rango epistémico, testimonios bien establecidos, entre otras cosas. Así, podemos encontrar en el ensayo "Del auge y progreso de las artes y las ciencias" un claro ejemplo de la estructura metodológica propuesta por Hume, donde los casos puntuales a partir de los cuales se establece la afirmación general son hechos históricos. Generalmente, Hume acompaña la mención de esos hechos con una nota al pie donde se amplían y se menciona la fuente de la cual provienen. En este ensayo se busca demostrar que las artes y ciencias no surgen y se desarrollan a consecuencia del genio, gusto e iniciativa de unos pocos, sino que son el resultado de las condiciones sociales y culturales de un pueblo. Para dar sustento a esta 
afirmación, Hume formula cuatro enunciados generales, los que a su vez se erigen a partir de hechos históricos concretos.

El primer enunciado consiste en que el las artes y las ciencias sólo prosperan en el marco de un gobierno libre antes que en una monarquía absoluta, debido a que, en esta última, no se gobierna mediante leyes sino que impera la voluntad arbitraria e ilimitada del soberano, mientras que en la primera, las leyes brindan un marco de seguridad que genera las condiciones para el surgimiento de la curiosidad y el ulterior desarrollo del conocimiento. Este enunciado se apoya en casos concretos como el del zar Pedro I de Rusia para el caso de la monarquía, y el del decenvirato de la antigua Roma que limitó el poder de los cónsules, para el de la república. El segundo enunciado consiste en que el desarrollo de las artes y ciencias se ve estimulado si se da en el contexto de un conjunto de Estados vecinos independientes, lo que evita que impere por mucho tiempo una única escuela de pensamiento o artística, antes que en un territorio extenso gobernado por un solo príncipe, que es más propenso a incurrir en excesos de autoridad y poder. En este último, la distancia alimenta la veneración infundada hacia el monarca, la superstición y la ignorancia, mientras que en los estados pequeños se favorece el razonamiento y el aprendizaje mutuo mediante la comparación y la evaluación que generan el intercambio de obras de arte. Como ejemplo que apoya esta última situación, Hume menciona a la antigua Grecia, conformada por una serie de pequeños Estados con un intercambio fluido entre ellos, y como ejemplos de la situación opuesta menciona a la Iglesia católica, que se convirtió en una suerte de gran Estado en el medioevo, donde sólo se admitía la filosofía peripatética, y el vasto imperio chino. El tercer enunciado propone que si bien las artes y las ciencias surgen en el marco de gobiernos republicanos, pueden luego transplantarse a Estados con otros regímenes, lo que habilita a decir que las ciencias prosperan mejor en una república, mientras que las artes cultas lo hacen en una monarquía. Esta afirmación surge de la observación de que las reglas de urbanidad o cortesía, que son parte de las "artes de la conversación" son más evidentes en los Estados europeos bajo regimenes monárquicos que en las repúblicas, mientras que no ocurre lo mismo con el desarrollo de las ciencias, debido a la "veneración supersticiosa de sacerdotes y príncipes," que reduce "por lo común, la libertad de razonamiento" (ESY - RP, 126 [p. 139]). Lo mismo sostiene que se corrobora en la antigüedad clásica, donde nota en autores de la talla de Cicerón, Horacio u Ovidio, una licenciosidad llamativa. Pou último, el cuarto enunciado afirma que, una vez que las artes y las ciencias alcanzan la 
perfección en un Estado, comienzan a declinar y raramente renacen en ese mismo lugar. Esto se debe a que frente a modelos de excelencia como un Homero o un Virgilio, es muy difícil para un joven artista proponer algo que los supere. Lo mismo evidencia, por ejemplo, la importación de pintura italiana a Inglaterra, que inhibió el desarrollo de este arte en las Islas Británicas, o la difusión en Alemania de obras literarias francesas, que frenaron el desarrollo de este arte en la propia lengua germánica.

En los ensayos y otros textos breves es donde más claramente pueden discernirse las etapas del método, que en los escritos más largos suelen perderse de vista a raíz de la interposición de digresiones o consideraciones teóricas. Así, encontramos una estructura similar a la que acabamos de presentar en ensayos tales como "De la superstición y el entusiasmo," "De lo populoso de las naciones antiguas" o "De los caracteres nacionales," por mencionar algunos. Por último, nos parece también claro el modo en que el método experimental aparece en Historia natural de la religión, escrito breve que originalmente fue publicado como una de las Cuatro Disertaciones. Por razones de espacio, nos concentraremos en mostrarlo sólo en relación con la cuestión del origen monoteísta o politeísta de la religión.

En esta obra, Hume investiga cuál es el origen del sentimiento religioso. Contrariamente a lo que afirmaban teólogos y filósofos de su época, sostiene que proviene de pasiones indirectas como la esperanza y el temor, antes que consistir en un instinto originario y, por ende, universal. En consonancia con este inusual punto de vista, considera que las religiones primitivas de la humanidad fueron politeístas y no monoteístas, dado que la civilización se ha ido desarrollando desde estadios primitivos y rudimentarios hacia otros más refinados, cada uno de los cuales se asocia con uno de los tipos de religión en cuestión. Esta afirmación se sustenta en una observación de carácter general: los registros históricos muestran unánimemente que "es una cuestión de hecho incontestable que hace unos mil setecientos años la humanidad en su conjunto era politeísta" (NHR 1.40 [p. 41]). Inclusive, en la actualidad, Hume observa que "tribus salvajes" de América, África y Asia, que se encuentran, por así decir, en la infancia de la civilización, son politeístas. En segundo lugar, sostiene que las primeras ideas religiosas no surgieron de la contemplación de las obras de la naturaleza, que evidencian un designio y regularidad dignos de un único autor inteligente, sino de los acontecimientos y afecciones corrientes de la vida humana. Esto se apoya en el hecho de que las deidades de las religiones politeístas dirigen y protegen cada acción humana: el matrimonio, el 
nacimiento, el cultivo de la tierra, la navegación, la guerra, tal como lo documentan Plinio o Hesíodo. Esas deidades, además, se caracterizan como imperfectas, más cercanas a los hombres que las adoran que a los seres ominipotentes y omniscientes que proponen las religiones monoteístas. Esto puede observarse tanto en China, como surge del relato del misionero Louis Leconte, como en la antigua Grecia y Roma, tal como Jenofonte, Suetonio o Quinto Curcio lo testimonian. Hume sostiene, además, que, a raíz del carácter imperfecto que se les adscribía, no se consideraba que las deidades de las religiones politeístas fueran las creadoras del universo, sino que es una cuestión que permanecía en suspenso o era reservada a los filósofos, tal como queda expresado en las obras de Ovidio, Diodoro Sículo o Sexto Empírico. Luego concluye que "casi todos los idólatras, de cualquier época o país, coinciden en estos principios e ideas generales, e incluso las características y dominios que asignan a sus deidades no son muy diferentes" (NHR 5.78 [p. 79]).

Inclusive al pasar al estudio del origen del monoteísmo, Hume sostiene que, en un comienzo, la idea de un único ser perfecto, creador del mundo, no surge del razonamiento, sino de la adulación y la necesidad de encontrar sentido a acontecimientos excepcionales tales como catástrofes naturales, epidemias, etc., antes que de la admiración del orden y regularidad que se observa en cada parte del universo.

Si bien podemos notar que el orden de la exposición en los ejemplos que acabamos de revisar suele ir de la afirmación de carácter más general a otras de menor generalidad que la apoyan y por último exponer los hechos en las que se basan, esto no debe llevar a confundir el método con el de la "ciencia abstracta" que mencionamos antes (vd. EPM 1.10), porque no sigue un orden lógico, sino que contradice muchas veces lo que, valiéndonos sólo de la razón "teórica" y no de la experiencia, puede pensarse que es lo que ha ocurrido. El orden que impone la sola razón no necesariamente coincide con lo que sucede de hecho y eso es lo que se manifiesta por medio del método experimental. El hecho de que el monoteísmo sea el tipo de religión más perfecto desde un punto de vista racional, en tanto tiene mayor poder explicativo porque da cuenta de la mayor cantidad de fenómenos mediante la menor cantidad de causas, que a su vez son las más simples y perfectas, no es razón suficiente para pensar que fue la primera religión del hombre que luego se "corrompió" en politeísmo, porque una multitud de hechos indican lo contrario. Entonces se vuelve necesario proponer una explicación que esté en consonancia con esos hechos. Esto es lo 
que el método experimental viene a ofrecer, y lo que marca la gran diferencia entre el ámbito de las cuestiones de hecho y el de las relaciones de ideas.

\section{Conclusiones}

Este recorrido por algunos tópicos de la obra de Hume ha tenido por objeto ayudar a esclarecer ciertos aspectos relevantes inherentes a su vinculación con la filosofía experimental. En particular, hemos contribuido a forjar una concepción de su método que excede los estereotipos del mentalismo y la introspección con los que suele asociarse la concepción del conocimiento de la Filosofía Moderna, al mostrar que el objeto de estudio de la ciencia de la naturaleza humana incluye pero excede el ámbito de los contenidos mentales del investigador para abarcar la conducta e interacción sociales. Por otra parte, hemos mostrado que la filiación de Hume con el movimiento experimentalista -que va más allá del newtonianismo- permite comprender de manera cabal su propia metodología y el énfasis que pone en la relevancia de los hechos, el rechazo de las especulaciones abstractas y el mantenerse dentro de los límites de las capacidades cognoscitivas humanas. Finalmente, consideramos haber esclarecido cuál es el fundamento que justifica la aplicación del método experimental a los asuntos morales, que está ligado a aspectos de índole ontológica, dado que supone que tanto los fenómenos naturales como los morales forman parte de las cuestiones de hecho. Esto amerita que puedan ser tratados de la misma manera, tanto respecto del modo en que se establecen los hechos - a pesar de que los de tipo moral no sean pasibles de experimentación-, como respecto de los principios que podemos establecer a partir de ellos a través de la reducción explicativa. 


\section{Bibliografía}

Obras de David Hume (en todos los casos la paginación de las traducciones al español aparece entre corchetes)

Hume, D. A Treatise of Human Nature. (T) Editado por David Fate Norton y Mary J. Norton. Oxford: Oxford University Press, 2007. Traducido por Felix Duque, Buenos Aires: Orbis, 1984. Citado por número de libro, parte, sección y paginación de la edición canónica de Selby-Bigge precedida por las iniciales SB.

Resumen del Tratado de la naturaleza humana. (ABST) Edición bilingüe de José L. Tasset. Barcelona: Literatura y Ciencia/Montesinos, 1999. Citado por la paginación de la edición canónica de Selby-Bigge precedida por las iniciales SB.

An Enquiry Concerning Human Understanding. (EHU) Editado por Tom L. Beauchamp. Oxford: Oxford University Press, 1999. Traducido por Jaime Salas Ortueta. Madrid: Alianza, 1980. Citado por el número de sección y el número de párrafo.

An Enquiry Concerning the Principles of Morals. (EPM) Editado por Tom L. Beauchamp. Oxford: Oxford University Press, 1998. Traducido por Marcelo Mendoza Hurtado. Bernal: Universidad Nacional de Quilmes/Prometeo, 2015. Citado por número de sección y número de párrafo.

History of England, 6 vols. (HE) Indianapolis: Liberty Fund, 1983.

Citado por del número de volumen, número de capítulo y página.

The Natural History of Religion/Historia natural de la religion. (NHR) Edición bilingüe. Introducción de Sergio Rábade. Traducción de Concha Cogolludo. Madrid: Trotta, 2003. Citado por número de sección y página.

"Of the Passions." (OP) Four Dissertations and Essays on Suicide and the Immortality of the Soul. Introducción de John Immerwahr (Four Dissertations) y John Valdimir Price (Essays on Suicide and the Immortality of the SouD), prefacio de James Fieser. Indiana: St. Augustine's Press, 2000. 
Traducido por José Luis Tasset Carmona. Barcelona: Anthropos, 2004, pp. 73-153. Citado por número de sección y página.

Essays Moral, Political, Literary. (ESY) Editado por Eugene F. Miller. Indianapolis: Liberty Fund, edición revisada, 1987 (1a ed., 1985). Traducido por Carlos Martín Ramírez. Madrid: Trotta/Liberty Fund, 2011. Citado por las iniciales del título del ensayo, seguidas de la paginación de la edición de Miller. Los ensayos utilizados son: "Of the Independency of Parliament" (IP), "Of Civil Liberty" (CL), "Of Commerce" (C), "Of Money" (M), "Of Interest" (I), "Of the Balance of Trade" (BT), "Of the Populousness of Ancient Nations" (PA), "Of Suicide" (Su), "Of the Immortality of the Soul" (IS), "Of the Rise and Progress of the Arts and Sciences" (RP).

Dialogues concerning Natural Religion. (DNR) Edición e introducción de Norman Kemp Smith. Indianápolis: Bobbs-Merrill, 1947. Traducido por Carlos Mellizo. Buenos Aires: Aguilar, 1981 (1ª. ed., 1973). Citado por número de parte y paginación de la edición de Kemp Smith.

The Letters of David Hume, 2 vols. (L) Editado por J. Y. T. Greig. Oxford: Clarendon Press, 1932. Citado por volumen, número de carta y número de página. 


\section{Bibliografía secundaria}

Anderson, R. F. "The Location, Extension, Shape and Size of Hume's Perceptions." En S. Tweyman, S. (ed.) (1995), pp. 153-167.

BIENER, Z. y SCHLIESSER, E. (eds.) Newton and Empiricism. Nueva York: Oxford University Press, 2014.

BoeHm, M. “Hume's Foundational Project in the Treatise." European Journal of Philosophy, 24, pp. 55-77, 2013.

BroAdie, A. (ed.) The Cambridge Companion to the Scottish Enlightenment. Cambridge: Cambridge University Press, 2003.

"The Human Mind and his Powers." En A. Broadie (ed.) (2003), pp. 62-63.

Broughton, J. “The Inquiry in Hume's Treatise." The Philosophical Review, 113, pp. 537-556, 2004.

"What Does the Scientist of Man Observe?" Hume Studies, 18, pp. 155-168, 1992.

BuCKLE, S. Hume's Enlightenment Tract. The Unity and Purpose of An Enquiry Concerning Human Understanding. Nueva York y Londres: Oxford University Press, 2001.

CARLIn, L. The Empiricists: A Guide for the Perplexed. Londres: Continuum, 2009.

Chambers, E. A Cyclopaedia or, An Universal Dictionary of Arts and Sciences. Londres, 1728.

CHAUVIN, E. Lexicon philosophicum. Leeuwardiae, 1692.

CRANE, T. "Mental Causation." Proceedings of the Aristotelian Society Supplementary Volumes, 69, pp. 211-253, 1995.

Cummins, P. "Hume as a Dualist and Anti-Dualist." Hume Studies XXI, pp. 47-55, 1995.

DE PIERRIS, G. "Locke and Hume on Scientific Methodology: The Newtonian Legacy.” Hume Studies, 32, pp. 277-330, 2006. 
DEAR, P. “The Meaning of Experience.” En K. Park y L. Daston (eds.) (2006), pp. 106-131.

Demeter, T. "Enlarging the Bounds of Moral Philosophy. Newton's Method and Hume's Science of Man.” En Z. Biener y E. Schliesser (eds.) (2014), pp. 171-204.

"Hume's Experimental Method." British Journal for the History of Philosophy, 20, pp. 577-599, 2012.

Dicker, G. Hume's Epistemology and Metaphysics An Introduction. Londres: Routledge, 1998.

Dobre, M. y NYDEN, T. (eds.) (2013) Cartesian Empiricisms. Dordrecht: Springer, 2013.

y __ "Introduction." En M. Dobre y T. Nyden (eds.) (2013), pp.

$1-21$.

Everson, s. "The Difference between Feeling and Thinking." Mind, new series, 97, pp. 401-413, 1988.

FEINGOLD, M. “Experimental Philosophy': Invention and Rebirth of a Seventeenth-Century Concept." Early Science and Medicine, 21, pp. 128, 2016.

Flage, DANIEL. David Hume's Theory of Mind. Londres y Nueva York: Routledge, 1990.

FLEW, A. "Impressions or Experiences: Public or Private?”, Hume Studies, 11, pp.183-191, 1985.

Guerrero Del AMO, J. “El problema mente-cuerpo en Hume.” Thémata. Revista de filosofia, 26, pp. 55-65, 2001.

HaAkONSSEN, K. "The History of Eighteenth-Century Philosophy: History or Philosophy?” Knud Haakonssen (ed.) (2006), pp. 3-25.

"The Idea of Early Modern Philosophy." En J. B. Schneewind (ed.) (2004), pp. 99-121.

HaAkonssen, Knud (ed.) The Cambridge History of Eighteenth-Century Philosophy. Nueva York: Cambridge University Press, 2006. 
HAZONY, Y. "Newtonian Explanatory Reduction and Hume's System of the Sciences." En Z. Biener y E. Schliesser (eds.) (2014), pp. 138-170.

Heinemann, F. H. “The Analysis of 'Experience'.” The Philosophical Review, 50, pp. 561-584, 1941.

ImMERWAHR, J. "The Anatomist and the Painter: The Continuity of Hume's Treatise and Essays." Hume Studies, XVII, pp. 1-14, 1991.

Kemp smith, N. The Philosophy of David Hume. A Critical Study of its Origins and Central Doctrines. Hampshire: Palgrave McMillan, 2005 (1a ed. 1941).

MANZO, S. "Experimentación, instrumentos científicos y cuantificación en el método de Francis Bacon.” Manuscrito, XXIV, pp. 49-84, 2001.

Norton, D. F. y nORTON, M. J. "Annotations to the Treatise." En D. Hume (2007), pp. 423-565.

Noxon, J. Hume's Philosophical Development. A study of his methods. Londres: Oxford University Press, 1973.

Owen, D. Hume's Reason. Nueva York: Oxford University Press, 1999.

PARK, K. y DASTON, L. (eds.) The Cambridge History of Science, vol. 3: Early Modern Science. Cambridge: Cambridge University Press, 2006.

PASSMORE, J. Hume's Intentions. Cambridge: Cambridge University Press, 1952.

PITSON, A. Hume's Philosophy of the Self. Londres y Nueva York: Routledge, 2002.

RÁBADE ROMERO, S. "La noción de experiencia en el empirismo inglés: Hume.” Diálogos. Revista del Departamento de Filosofía de la Universidad de Puerto Rico, IX, pp. 33-51, 1973.

RIVERS, I. Reason, Grace and Sentiment. A Study of the Language of Religion and Ethics in England, 1660-1780. Volume II: Shaftesbury to Hume. Cambridge: Cambridge University Press, 2000.

RobisOn, w. "Hume and the Experimental Method of Reasoning." Southwest Philosophy Review, 10, pp. 29-37, 1994.

SCHLIESSER, E. "Hume's Attack on Newton's Philosophy." Enlightenment and Dissent, 25, pp. 167-203, 2009. 
SchneEwind, J. B. (ed.) Teaching New Histories of Philosophy. Nueva Jersey: University Center for Human Values, 2004.

SHAPIN, S. The Scientific Revolution. Chicago: The University of Chicago Press, 1998.

SHAPIRO, B. A Culture of Fact, 1550-1720: Ithaca, Cornell University Press, 2000.

Slavov, M. "Newtonian and Non-Newtonian Elements in Hume." The Journal of Scottish Philosophy, 14, pp. 275-296, 2016.

STROud, B. Hume. Londres y Nueva York: Routledge \& Keagan Paul, 1977.

SYTSMA J. y BUCKWAlter, w. (eds.) A Companion to Experimental Philosophy. Malden: Wiley-Blackwell, 2016.

TASSET, J. Estudio introductorio. En D. Hume (1999), pp. 3-108.

Tweyman, s. (ed.) David Hume: Critical Assessments. Londres y Nueva York: Routledge, 1995.

Wood, P. B. "The Natural History of Man in the Scottish Enlightenment." History of Science, 28, pp. 89-123, 1989. 